\title{
Preliminary study towards the definition of a PHM framework for the hydraulic system of a fly-by-wire helicopter
}

\author{
Andrea De Martin ${ }^{1}$, Giovanni Jacazio ${ }^{2}$ and Massimo Sorli ${ }^{3}$ \\ 1,2,3 Politecnico di Torino - Department of Mechanical and Aerospace Engineering, Torino, 10129, Italy \\ andrea.demartin@polito.it \\ giovanni.jacazio@polito.it \\ massimo.sorli@polito.it
}

\begin{abstract}
On-board hydraulic systems are tasked to provide a number of critical functions to ensure the in-flight operability of rotary-wings vehicles; the hydraulic system is needed to supply power to the flight control actuators and a number of other utilities, as well as to condition the hydraulic fluid, under a wide range of possible in-service conditions. Being a flight-critical system, the definition of a Prognostics and Health Management framework would provide significant advantages to the users, such as better risk mitigation and improved availability. Moreover, a significant reduction in the occurrences of unpredicted failures, one of the more known downsides of helicopters, is expected. A preliminary analysis on the effects of the inception and progression of several degradation types is the first step assess the feasibility of a PHM system for new platforms, and which failure modes are more likely to be observed. Further, since several key components are frequently provided by different suppliers to the airframer, this preliminary analysis would allow to better assess if an Integrated Vehicle Health Management approach, integrating signals coming from different components, could be beneficial. To pursue this study, a complete model of the hydraulic system for a flyby-wire helicopter has been prepared. Then, an in-depth simulation campaign was pursued with the aim of studying the interactions between different failure modes, the effects that the propagating degradations have on the system performances and which signals can be used to define a robust set of features. The paper introduces the case-study under analysis, a general configuration for fly-by-wire helicopters, presenting the most prominent peculiarities of the system and the effect of such peculiarities on the definition of health monitoring schemes. The model is then used to describe the behavior of the system under nominal and degraded conditions is introduced. Between the possible \footnotetext{
the terms of the Creative Commons Attribution 3.0 United States License, which permits unrestricted use, distribution, and reproduction in any medium, provided the original author and source are credited.
}

Andrea De Martin et al. This is an open-access article distributed under
\end{abstract}

failure modes, the interaction between wear in several mechanical components and the clogging of the hydraulic lines filters was chosen as the subject of this study; motivations are provided and the degradation model described in detail. Hence, results of a wide-ranging simulation campaign are presented, where the time-domain response of the system is used to guide in the definition of a proper set of features able to characterize the selected fault cases. Selected features are presented, chosen according to significant metrics such as correlation with the simulated degradations, signal-to-noise ratio and accuracy. Two different approaches with a varying degree of integration between system signals are proposed and compared. Prognostics is then pursued through well-known particle filter algorithms. The analysis provides promising results on the capability of successfully detecting, isolating and identifying the selected fault mode; laying the foundations for further and more comprehensive studies on the subject.

\section{INTRODUCTION}

Fly-by-wire electro-hydraulic flight control systems are an emergent technology for rotary-wing aircrafts, still widely adopting solutions based upon the well-established architecture made of hydraulic actuators with mechanical feedback and Stability and Control Augmentation Systems. Each actuator is usually supplied through two independent hydraulic circuits to meet the strict redundancy requirements needed to ensure the proper level of safety. The development of a PHM framework for the whole hydraulic system would provide significant advantages in terms of risk mitigation, availability and maintenance costs, which are particularly significant for rotary-wing aircrafts. The close dependence of the performances of each subsystem on the behavior of the other components of the whole system makes it important to prepare a comprehensive study, not limited to one component or assembly. The same close inter-dependence between components behavior and failures development also suggests that a more integrated approach to PHM is of interest. 
Although relevant to both safety and availability of the whole vehicle, literature on the subject is sparse and mostly related to the analysis of one isolated component. On similar matters for fixed-wing aircraft, (Byington et al. 2004) presented a work on the possibility of developing a PHM system for the F/A-18 stabilizer Electro-Hydraulic ServoValves (EHSVs). (Adams et al. 2016; Brown et al. 2009) presented different approaches to the PHM of hydraulic actuators. A work on the feasibility of a PHM system for the Electro-Hydraulic Servo Actuator of a wide-body aircraft was described in (Autin et al. 2018), while a preliminary study on the possibility to perform PHM functions on the SCAS actuator of a traditional flight control system for helicopter is described in (Nesci et al. 2020). A few works adopting a more system-wide approach focused on diagnostic issues were provided by (Smith et al. 2014) and (Vianna and Malere 2014). Authors of this paper performed a preliminary study over the PHM capabilities of a single Power Control Module for rotary-wing applications in (De Martin et al. 2019); this early study was based on a simplified model of the system and was aimed at the early definition of features and PHM technologies upon which this paper expands. The paper is structured as follows. At first, the case study is presented and discussed in detail, highlighting the available sensors and the operative conditions of particular interest for PHM activities. Hence, the dynamic model of the whole system is introduced and discussed, as well as the embedded models for the selected degradation processes. Results from simulation campaigns are hence used to perform a preliminary study on different PHM solutions, which performances are hence analyzed and compared.

\section{Case Study}

The system chosen as case study for the presented research activities is the hydraulic system of a helicopter with fly-bywire flight control actuators. As depicted in Fig. 1, the system can be roughly divided in three major subsystems. Two independent variable displacement axial-piston pumps supply fluid to the actuation system by means of two separate Power Control Modules (PCMs). The actuation system is provided with four tandem Electro-Hydraulic Servo Actuators (EHSA), three controlling the collective and cyclic command to the main rotor and one acting on the tail rotor. Each actuator is connected to two independent hydraulic lines, each managed by one PCM. The architecture of each PCM is derived from the one described in (De Martin et al. 2019) and reported in Fig.2. A highpressure, variable displacement pump is used to convey the oil from the pressurized tank reservoir to the user; the fluid must pass through a check-valve used to avoid backflow issues and is forced through the high-pressure filter (1). A pressure relief valve (2) is inserted in derivation to avoid overpressures and a circuit shutoff valve (3a) is used to eventually disconnect the flight control users (Main Rotor
Actuators, MRA and Tail Rotor Actuators, TRA) from the PCM supply line. A utility shutoff valve (3b) provides the same functions to the hydraulic line connected with the accessories. A low-pressure filter (4) is put on the return line, which brings back the oil to the reservoir (5). The system comes equipped with a set number of sensors, most of which provides only a digital on/off output; to this category belong the pressure switches (6) positioned on the supply line to provide alert in case of failure conditions, and the oil level indicators mounted on the reservoir (7). The system is then equipped with a pressure transducer on the supply line (8), differential pressure indicators on the filters and an oil temperature sensor situated in the reservoir (9). Not shown, fuel-oil heat exchangers are used to keep the oil temperature under dangerous thresholds. Each EHSA can be described through the functional scheme depicted in Fig.3, although geometrical dimensions can vary between the Main Rod Actuators (MRAs) and the Tail Rod Actuator (TRA). Each cylinder is supplied by each hydraulic line through dedicated servo-valves $(1 \mathrm{a}, 1 \mathrm{~b})$ with doubleelectrical redundancy. LVDTs are used to measure the actuators position and close the control loop at the actuator level (2); pressures in each chambers of the actuator are monitored. The position of each servovalve spool is also measured through dedicated LVDTs for monitoring purposes, while the value of the servovalve currents is known through the control loop. The PCMs distributing the hydraulic fluid flow to the helicopter actuators and utilities are normally equipped with sensors used to enable some diagnostics of the hydraulic system.

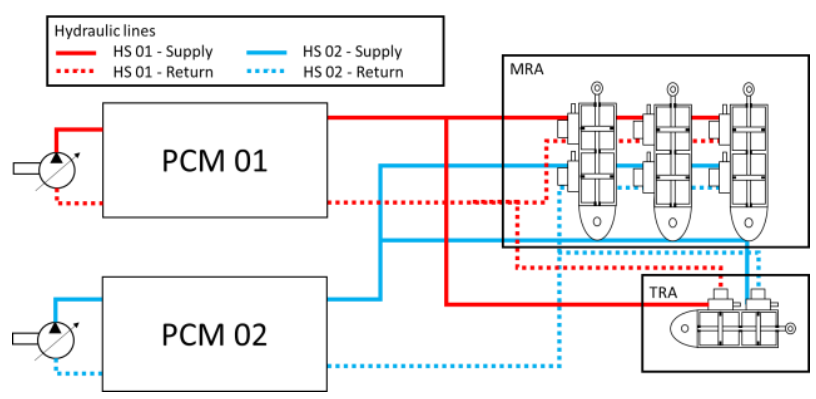

Fig. 1.: Case study architecture

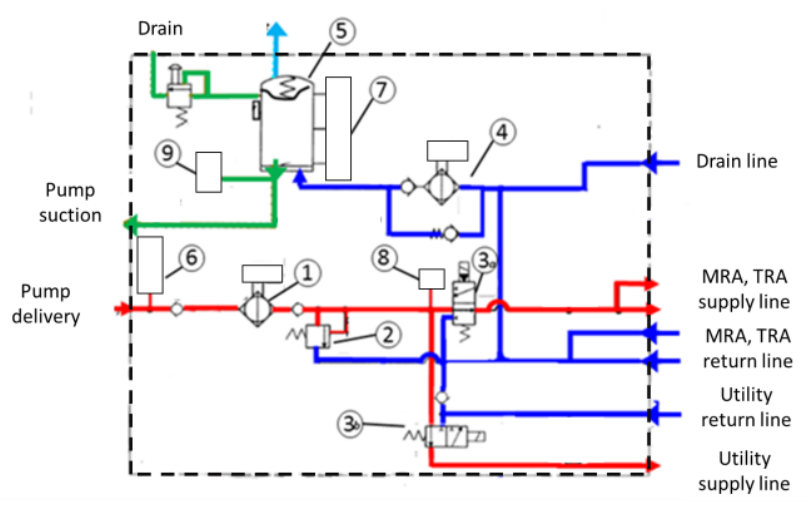

Fig.2: PCM architecture 


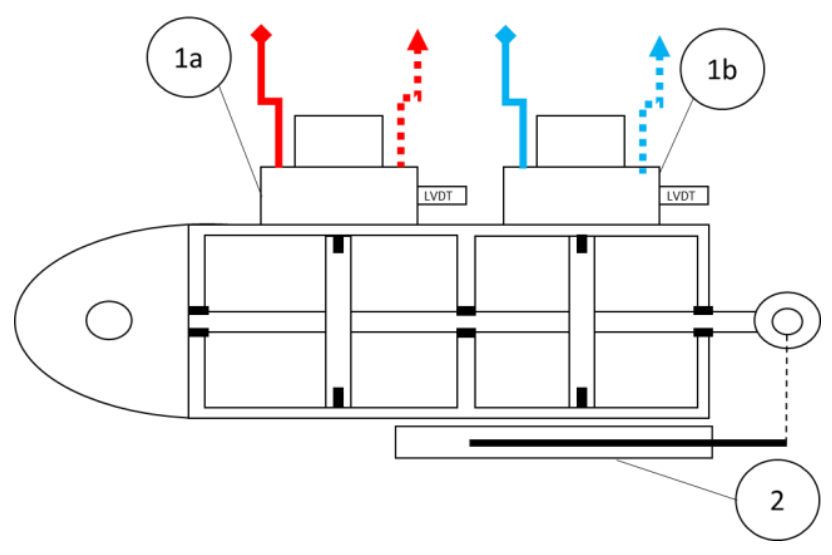

Fig.3: Schematics of the tandem EHSA

These measuring devices normally are:

- Oil level switches in the reservoir to signal full, low and empty conditions for the reservoir

- Visual indicator of oil level in the reservoir

- Sensor measuring the pressure in the pump discharge line

- Differential pressure indicators measuring the pressure drop across the filters, normally provided with a thermal lockout switch disabling the operation at low oil temperatures, hence preventing triggering false alarms

- Temperature sensor for measuring the oil temperature in the reservoir

In some helicopters, differential pressure sensors are located in the pump drain lines to infer the value of the pump drain flow by measuring the pressure differential across an orifice placed on the drain line. It must be noticed, however, that the information obtained by all these sensors is used in the existing hydraulic systems for helicopters by signaling a failure, and not for performing system prognostics. In particular, the information of the differential pressure across the filters is normally provided by differential pressure switches giving an on/off indication. Also, the oil level condition in the reservoir is normally a discrete information generated by switches, and not by a sensor providing a continuous measurement. The objective of the work described in the paper is primarily to fuse together all available information, with a possible addition of a minimum number of other sensors, in order to move from a simple diagnostic system indicating healthy / faulty status, to a diagnostic and prognostic system capable of better disambiguating faults and of allowing the implementation of prognostic functions.

\section{SYSTEM MODEL}

Following the approach already used by authors for a similar research on EHSAs for fixed-wing aircraft (Autin et al. 2018), a high-fidelity model of the system was prepared and implemented in Matlab/Simulink. To best characterize the system behavior and its dependence on widely varying operating conditions the vast majority of the components have been modelled according to detailed physics-based equations, while the characteristics of the hydraulic fluid are described as function of the simulated temperature and airfraction.

\subsection{Variable-displacement axial-pistons pump}

The axial pistons pump supplying each hydraulic line is a nine-pistons, variable displacement device. Driven directly by the helicopter's engine through its gearbox it is used to transform part of the mechanical power produced by the propulsion system into hydraulic power then used by the several actuators present on board. The axial pistons solution with variable displacement is currently the most adopted in PCM form on-board duty, since it provides consistent performances in high-pressure environments and allows to obtain higher efficiency by limiting the supplied flow-rate to the strictly necessary, avoiding the need of an always metering pressure relief valve. Calling $Q_{i}$ the net flow-rate leaving the $\mathrm{i}$-th piston, the total flow-rate of the pump can be computed as,

$$
\mathrm{Q}=\sum_{i=1}^{N} Q_{i}-Q_{\text {leak }}
$$

Where $Q_{\text {leak }}$ is the total flow-rate lost due to leakages between the rotating pistons, the swash plate and the pump case. Neglecting at first the fluid compressibility $\beta$ and modelling the leakages as purely laminar flows, Eq. (1) can be rewritten as,

$$
Q=\omega \sum_{i=1}^{N} \frac{d V_{i}}{d \alpha}-K_{\text {leak }} \Delta P_{p}
$$

Where $\omega$ is the pump rotational speed, $V_{i}$ is the volume of each piston and $\alpha$ the angular position of the pump shaft. $K_{\text {leak }}$ is the constant of the Hagen-Poiseuille Law, while $\Delta P_{p}$ is the overall pressure drop across the pump.

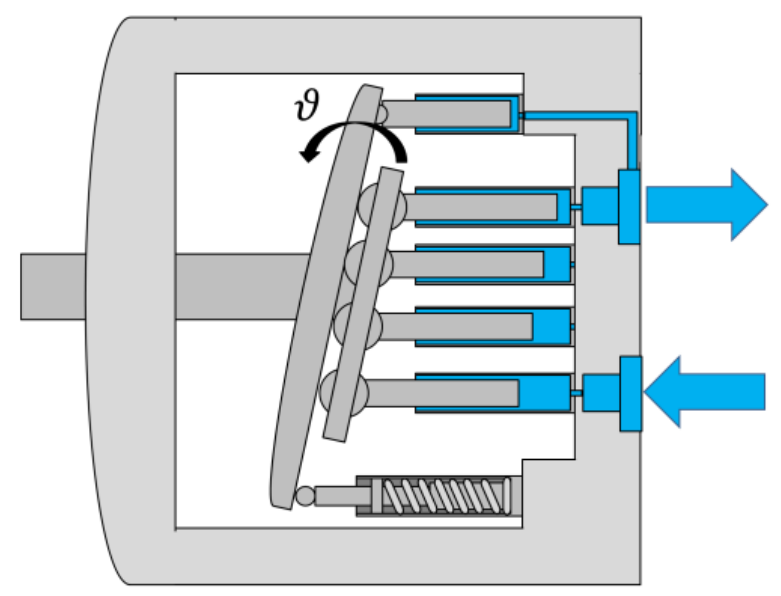

Fig.4. Scheme of a variable displacement axial pistons pump 
The supply pressure can hence be obtained by integrating the continuity equation across the whole pump and taking into account the fluid compressibility. The regulation of the pump displacement is also physically modelled; assuming a turbulent flow in the inlet $\left(Q_{\text {in }}\right)$ and outlet $\left(Q_{\text {out }}\right)$ ports of the regulating valve, not shown in Fig.4, it is possible to determine the pressure as,

$$
\frac{d P_{c}}{d t}=\frac{\beta}{V}\left(Q_{\text {in }}-Q_{o u t}-\frac{d V}{d t}\right)
$$

The mechanical equilibrium of the swashplate can hence be written as,

$$
P_{c} A_{C} R_{c}=I_{p} \ddot{\vartheta}+\gamma \dot{\vartheta}+k R_{c}\left(R_{c} \vartheta\right)
$$

Where $A_{C}$ is the cylinder area and $R_{c}$ is the leverage length. $\vartheta$ and $I_{p}$ are respectively the swash plate tilt angle and the associated inertia, while $\gamma$ and $k$ are the viscous friction coefficient and the spring stiffness. Since the displacement, and hence the supplied flowrate, is directly proportional to the tilt angle of the swashplate $\vartheta$ and defining as its maximum value $\vartheta_{\max }$, the instantaneous flow-rate supplied by the pump can be re-written as follows.

$$
Q=\omega \frac{\vartheta}{\vartheta_{\max }} \sum_{i=1}^{N} \frac{d V_{i}}{d \alpha}-K_{\text {leak }} \Delta P_{p}
$$

\subsection{Power Control Modules}

Each hydraulic line is modelled as series of hydraulic resistances and hydraulic capacities; due to the length of the pipes linking the PCM with the actuation system, and especially with the Tail Rotor Actuator, distributed losses are not negligible and are computed through the Darcy Law. Although the geometry of the hydraulic lines is not known and depends on the helicopter design, localized pressure drops and other geometry variations have been included through reasonable hypothesis and computed according to (Idelchik and Fried 1986). Check-valves, no-back valves pressure relief valves have been physically modelled through varying declination of equations (6) and (7), where $C_{d}$ is the discharge coefficient function of the local Reynolds' number, $A$ is the metering passage area dependent on the valve lift $x$ and $\Delta P_{v}$ is the pressure drop across the poppet.

$$
\begin{aligned}
& Q_{v}=C_{d}(R e) A(x) \sqrt{\frac{2 \Delta P_{v}}{\rho_{h}}} \\
& m_{v} \ddot{x}+c_{v} \dot{x}+k_{v} x+F^{*}=\Delta P_{v} A_{v}
\end{aligned}
$$

The discharged flow-rate $Q_{v}$ also depends on the fluid density $\rho_{h}$, mostly function of the oil temperature. The valve lift can be computed through equation (7) and is limited to a maximum value defined by the device geometry; its behavior depends on the mechanical equilibrium defined by the force generated by the pressure drop across the metering edge $\Delta P_{v}$ and the section $A_{v}$, the poppet mass $m_{v}$, the viscous friction $c_{v} \dot{x}$ and the effect of the spring stiffness $k_{v}$ and pre-load $F^{*}$, whenever a spring is present. The reservoir of each PCM is also physically modelled as a spring-pressured tank. Filters have been modelled as a network of hydraulic resistances (De Martin et al. 2019); the total pressure drop across the filter $\Delta P_{f}$ can be thought as the sum of the pressure drop across the inlet and outlet interfaces $\Delta P_{f, \text { in }}$ and $\Delta P_{f, \text { out }}$, and the pressure drop across each layer due to localized $\Delta P_{f, L}$ and distributed losses $\Delta P_{f, D}$.

$$
\Delta P_{f}=\Delta P_{f, \text { in }}+\Delta P_{f, \text { out }}+\Delta P_{f, L}+\Delta P_{f, D}
$$

Parameters pertaining each pressure drops have been fitted over experimental data sets obtained for nominal conditions.

\subsection{Actuation system}

The model of each actuator, represented in Fig.5, is derived from those described by authors in (De Martin et al. 2018) and employed in the feasibility analysis reported in (Autin et al. 2018). The same degradations described in (Autin et al. 2018) for the servovalves are modelled and can be simulated at any given time, including crack or backlash occurrence at the connection between the servovalve spool and its feedback spring, distortion of the jet pipe, clogging of the hydraulic amplificatory channels and degradation of the permanent magnets of the torque motors. A few key differences are worth highlighting. For once, each device is a tandem actuator, meaning that the behavior of two servovalves and four actuator's chambers are represented for each cylinder. The possibility to move the reference system $y_{h}$ integral with the helicopter frame is also introduced, allowing to describe the effects of the heavy vibratory environment on the actuators behavior, as well as on the inception and growth of faults in its components. As described in Fig.5, leakage paths are also included in the model, where they are described as the sum of two components. The first, represented through the HagenPoiseuille Law, models the presence of laminar flow between two adjacent chambers. The second component, based upon a small-orifice equation similar to Eq. (6), models the presence of localized defects or irregularities of the sealings. Friction at the contact points between the sealing components and the cylinder sleeve is computed according to (Frölich et al. 2014). The resultant dynamic equations for the cylinder rod and the cylinder sleeve hence become

$$
\begin{aligned}
& \left(P_{1} A_{1}-P_{2} A_{2}\right)+\left(P_{3} A_{3}-P_{4} A_{4}\right)-\sum F_{f r, i}-F_{\text {ext }} \\
& -W_{r}=m_{r} \ddot{x}+c \dot{x} \\
& k_{h}\left(y_{h}-y\right)+c_{h}\left(\dot{y}_{h}-\dot{y}\right)-\left(P_{1} A_{1}-P_{2} A_{2}\right) \\
& -\left(P_{3} A_{3}-P_{4} A_{4}\right)-\sum F_{f r, i}-W_{s}=m_{s} \ddot{y}+c \dot{y}
\end{aligned}
$$

Where the terms $W_{s}, W_{r}$ are the weights respectively of the sleeve and of the rod, to be considered for the MRAs due to their vertical mounting position. 


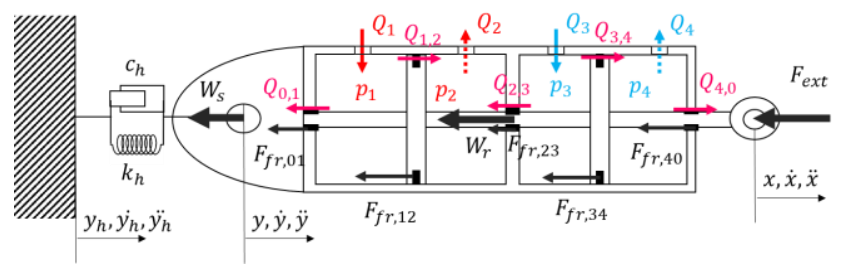

Fig.5. Fluid and mechanical model of the employed EHSAs.

\subsection{Main Rotor swash plate}

The variation of the collective or cyclic command acting on the main rotor is obtained by imposing similar or differential commands to each of the three actuators acting on the swash plate. As depicted in Fig.6, the swash plate is then modelled as its own dynamic system, connected to each actuator through their rod-ends, each with its own stiffness and damping. It becomes hence possible to compute the overall movement of the swash plate and express its position in terms of collective response and Eulerian angles.

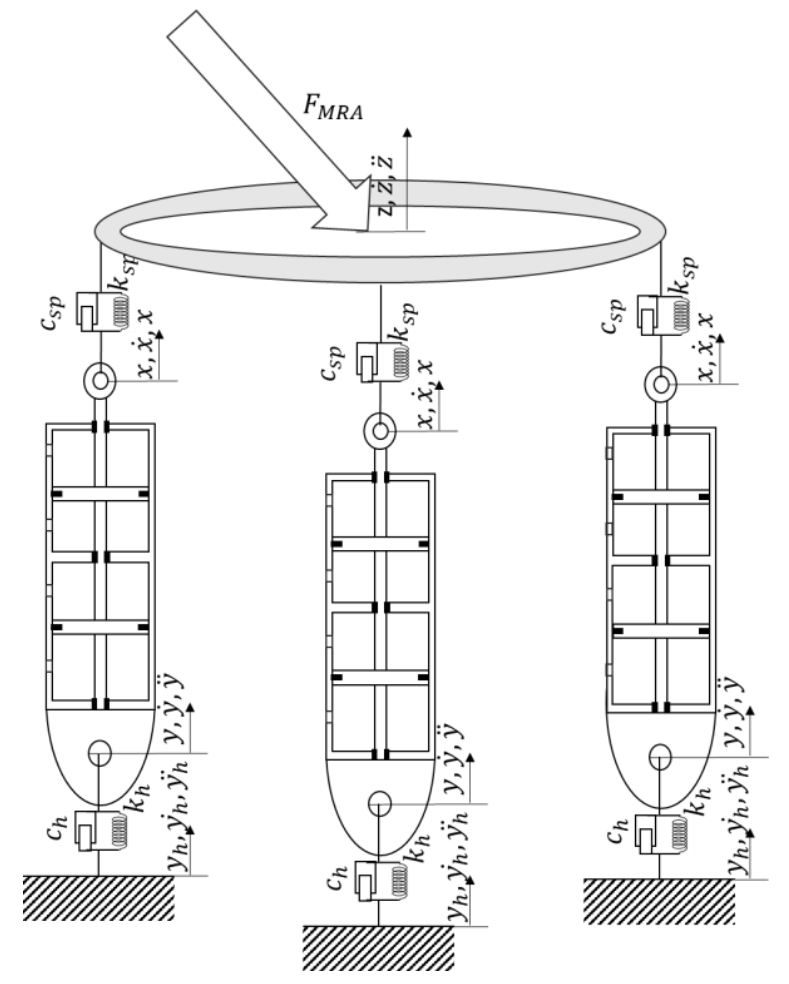

Fig.6. Scheme of the swash plate model.

\subsection{Hydraulic fluid behavior}

As previously mentioned, the properties of the hydraulic fluid are not kept constant during the simulations, but are free to change based on variation in the fluid temperature and air-fraction percentage according to the curves provided by the hydraulic fluid supplier. The temperature of the hydraulic fluid is computed within the simulation through a simplified thermal model based on lumped parameters. The power lost in each hydraulic line due to hydraulic resistances is continuously computed and used within the simplified model considering a constant flow-rate of fuel within the fuel-oil heat exchanger and an external temperature defined by the mission profile.

\section{DEGRADATION MODEL}

The degradation mode initially considered for the study is the possible clogging of the high-pressure and low-pressure filters due to wear in the actuators' seals and in the pumps' pistons. This degradation mode was chosen for two main reasons. For once, it is a fault mode which is directly caused by the degradation of other components. Secondly, it occurs within a subsystem (the PCM) which is equipped with a low number of sensors. Both characteristics makes this degradation an interesting case for an IVHM approach, possibly making use of all of the sensors employed within the hydraulic and flight control system.

\subsection{Wear of the actuators seals}

Each actuator is equipped with at least five sealing elements; two isolate the actuator from the outside environment, while the other three are positioned between adjacent chambers. During exercise, seals are pressed against the two elements in relative motion and are subjected to widely-varying spectrum of loads and relative speed. Although seals can be affected by a significant number of failure modes, such as opening of cracks and fatigue issues, abrasive wear due to repeated rubbing and passage of hard particles is still the most probable degradation. Addressing with $P_{s, i}$ the contact pressure of the $i$-th seal, almost proportional to the pressure between the two environment isolated by the seal itself, with $D_{s, i}$ and $W_{s, i}$ the seal diameter and its width, the worn volume due to relative motion at relative speed $v_{i}$, can be computed for the $i$-th seal as,

$$
V_{w, i}=\frac{K_{w} v_{i}\left(P_{s, i} \pi D_{s, i} W_{s, i}\right)}{H_{i}}
$$

Where $K_{w}$ is the wear constant and $H_{i}$ the material hardness. Under the hypothesis of uniform wear across the contact area, leakages between the $i$-th and the $i+1$ environment separated by the seal can hence be computed as.

$$
Q_{i, i+1}=\frac{\left[\frac{V_{W, i}}{\pi D_{s, i} W_{s, i}}\right]^{3}\left(\pi D_{s, i}\right)}{12 \mu W_{s, i}}\left(P_{i}-P_{i+1}\right)
$$

A similar approach is used to compute wear in the piston pump.

\subsection{Filter clogging}

Material particles detached due to wear are conveyed along the hydraulic lines by the fluid and brought towards the 
filters situated in the supply and in the return channels. The volume of worn material generated within the actuators $V_{w, T M R}$ is hence forced through the low-pressure filter on the return line. Although variations on this configuration exist, the case study makes use of higher filtering capabilities in the high-pressure line. As such, the fraction of volume which particles' size $d_{j}$ is higher than the rated filtration level $\alpha_{L P F}$ are kept within the filter, while the rest keeps flowing towards the pump and the high-pressure line. Recalling as $\Delta V_{w, L P F}$ the increase of worn volume within the low-pressure filter at time $t$, this situation can be modelled as,

$$
V_{w, L P F}=\left.\Delta V_{w, L P F}\right|_{t}+\left.V_{w, T M R}\right|_{t, d_{i}<\alpha_{L P F}}
$$

The high-pressure filter hence operates on the remaining worn volume coming from the actuators and on the volume worn within the pump $V_{w, p}$. Addressing with $\alpha_{H P F}$ its rated filtration level, the model becomes

$$
\begin{aligned}
& \left.\Delta V_{w, H P F}\right|_{t}=\left.V_{w, p}\right|_{t, d_{i} \geq \alpha_{L P F}}+\left.V_{w, T M R}\right|_{t, d_{i}<\alpha_{L P F}}- \\
& \left.V_{w, T M R}\right|_{t, d_{i}<\alpha_{H P F}}
\end{aligned}
$$

It is also important to note that the volume occupied by the worn material within the filter can and will be different from the sheer computation provided above. Assuming debris of spherical geometries, the minimum portion of the filter volume filled with particles (or the minimum volume of the cylinder able to contain a certain volume of spheres) can be computed as the Kepler volume (Eker et al. 2016). The progressive volume increase of the filtered material, hence causes an increase in the pressure drop across the filter. The model makes use of the popular formulations by Ergun (Ergun 1952) and Carman (Carman 1997) through the modified expression provided by (Eker et al. 2016) which take into account information such the amount of contaminants $L_{c}$, its porosity $\epsilon$, mean diameter $D_{p}^{2}$ and sphericity $\phi$ of the particles, superficial speed $V_{s}$ and density of the fluid $\mu$.

$$
\Delta P_{f, c}=\frac{150 V_{S} \mu(1-\epsilon)^{2} L_{c}}{D_{p}^{2} \epsilon^{3}}+\frac{1.75(1-\epsilon) \rho V_{S}^{2} L_{c}}{\epsilon^{3} D_{p}}
$$

Considering all the other parameters as constants, both models defines a direct proportionality between the additional pressure drop across the gap $\Delta P_{f, c}$ and the product between the fluid density and the amount of contaminants $L_{c}$.

\section{SIMULATION OF HEALTHY AND DEGRADED CONDITIONS}

To study the system behavior under healthy and progressively more degraded conditions we performed a simulation campaign replicating several helicopter flights. The command, loads and operating conditions sent to each component during each simulated flight are assembled by attaching together segments of signals drawn from a pool of possibilities adapted from a real-case scenario. The drawing process is such to remain representative of the statistical distribution of the amplitude and type of both of command and load signals. In the same way, vibrations at the actuators level are adapted from design specifications of similar systems. Temperature at ground level is also drawn randomly, while its value scales with altitude as in (Autin et al. 2018).

\subsection{Hypothesis}

A few hypotheses are defined for the simulation campaign:

- The external seals separating the actuator chambers from the outside environment are not subject to wear (no external leakages).

- The parameters of the Archard's Law differ for each seal, and their value is selected from a Gaussian distribution around the nominal mean.

- In sake of simplicity, the degradation progresses can only occur in the hydraulic system HS01. As such, only the filters in the PCM 01 are affected by the clogging process.

- Only the pump pertaining the PCM 01 generates debris.

- The actuators seals and the PCM01 filters are the only component interested by degradation processes. Every other component works under the assumption of healthy conditions.

\subsection{Degradation effect on the actuators' performances}

The major result of the progressive wear of sealing material within the servo-actuator is the increase of the leakages between adjacent chambers, which enable a series of cascade-effect on the system behavior, partially dependent on the type of the adopted control law and on which control loops are present within the servosystem.

Fig.7 describes the behavior of the Main Rotor Actuator 01 (MRA01) in response to an accelerated wear simulation. As it can be observed, the macroscopic behavior in position is hardly affected by the propagating fault. Closer inspection however reveals, as shown in (Autin et al. 2018), that the lack of noticeable performance variations during the early stages of wear progression is due to the system reaction; the EHSAs controllers closing the position loops acts by increasing the current command sent to the servovalves, hence producing wider openings of their spool, to compensate for the loss of flow-rate due to leakages. If pressure sensors are used to observe the operating conditions of the actuators' chambers, it is possible to notice the progressive loss of the servosystem pressure gain, defined as the ratio between the pressure drop across two adjacent chambers and the spool position of the related servovalve. With respect to previous studies on EHSAs for fixed-wing aircraft, the effect is less noticeable due to the presence of four chambers acting on the same rod. 

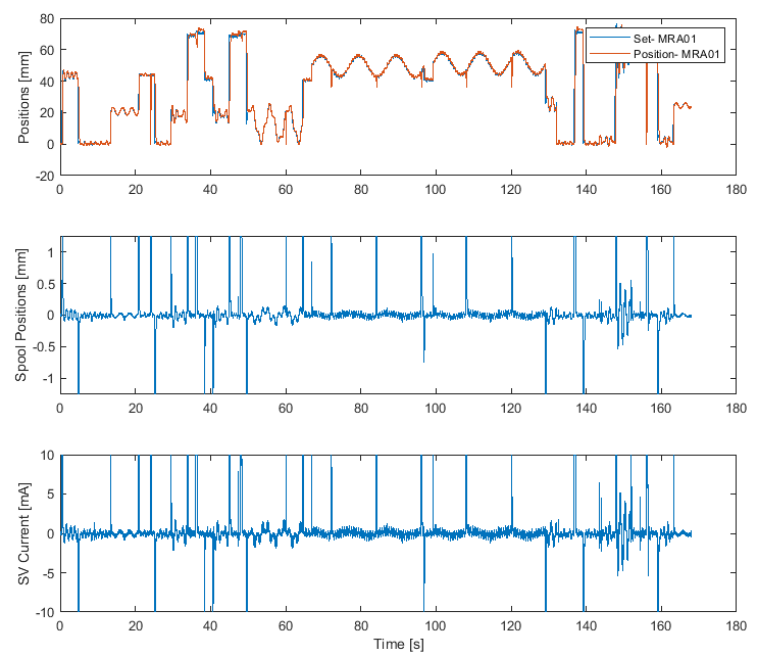

Fig.7. Leakage effect on actuators behavior.

\subsection{Degradation effect on the PCM behavior}

The progressive wear of several moving components, such as actuators seals and pumps cylinders, generates an increasing volume of worn material which begins to circulate within the hydraulic system. The portion of debris bigger than the filtration level of the filters is separated from the hydraulic fluid and kept within the filter that intercepted the particles first. In the system adopted as case-study, the most performing filter is the one mounted on the supplyline, which means that it has a high probability of early clogging with respect to the return-line filter. Hence, we focus our attention on this component. As defined through Eq.(15) and depicted in Fig.8, the most significant effect of the progressive deposit of more and more worn material is the increase of the pressure drop across the filter. As wellobserved by (Eker et al. 2016), the growth of such pressure drop is severely non-linear. The influence of temperature on the fluid viscosity, along with the irregular flow-rate request of the flight-control actuator, however makes this single symptom unsuitable as a prognostic feature.
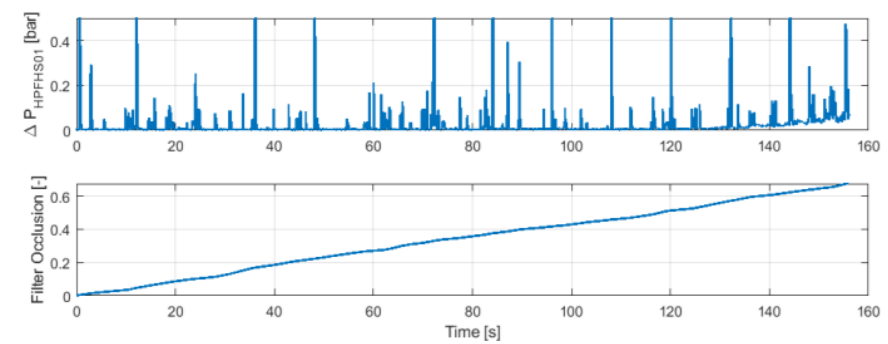

Fig.8. Degradation effect on the high-pressure filter behavior.

\section{PHM ACTIVITIES}

Health monitoring and prognostics for localized fault modes is pursued through the definition of features based upon signals coming from real or virtual sensors measuring physical quantities related to the evolving degradation. In literature, failure modes are typically studied as independent from one another, and introduced one at a time in simulation campaign or seeded tests. The degradation investigated in this paper is however the results of the interaction of several fault modes (wear of mechanical components, filter clogging) interesting different components within a complex system. The signals provided by the traditional configuration of PCM are not sufficient to successfully perform PHM activities (De Martin et al. 2019); although information related to the pressure drop across the filters and the oil temperature are simply obtainable, flow-rate signals measures are usually not available. At the same time, the clogging of the PCM filters is a direct product of the wear of other components, which sensors can carry information relevant to the leakage occurrence and hence to the volume of worn material, suggesting that moving towards an Integrated Vehicle Health Management approach could provide PHM capabilities without resorting to additional sensors. Two possible solutions with varying level of integration between on-board systems are studied and their performances compared. To compare the results of each solution, we decided to work with the same PHM architecture for fault detection and prognosis, based upon a combination of data-driven techniques and particle filtering.

\subsection{Fault detection through data driven approach}

The approach for fault detection is a purely data-driven one. A baseline representing the feature behavior under healthy conditions is built upon the first 100 data points and an automatic, customizable threshold is set to a value covering $95 \%$ of the baseline probability distribution. Simulation data are then streamed in and compared to the initial baseline; the anomaly detection flag is raised when the new distribution differs from the baseline by a customizable confidence level equal to or greater than $95 \%$. The fault detection is performed through a purely data-driven method, comparing the current feature distribution with a predefined baseline representative of the healthy conditions (Vachtsevanos et al. 2007).

\subsection{Failure prognosis}

The prognostic framework takes advantage of a nonlinear process (fault / degradation) model, a Bayesian estimation method using particle filtering and real-time measurements (Orchard and Vachtsevanos 2009). Prognosis is achieved by performing two sequential steps, prediction and filtering. Prediction uses both the knowledge of the previous state estimate and the process model to generate the a priori state pdf estimate for the next time instant,

$p\left(x_{0: t} \mid y_{1: t-1}\right)$
$=\int p\left(x_{t} \mid y_{t-1}\right) p\left(x_{0: t-1} \mid y_{1: t-1}\right) d x_{0: t-1}$ 
Unfortunately, this expression does not have an analytical solution in most cases. Instead, Sequential Monte Carlo (SMC) algorithms (Roemer et al. 2011) or particle filters, are used to numerically solve this equation in real-time with efficient sampling strategies. Particle filtering approximates the state pdf using samples or "particles" having associated discrete probability masses ("weights") as,

$p\left(x_{t} \mid y_{1: t}\right) \approx \widetilde{w}_{t}\left(x_{0: t}^{i}\right) \delta\left(x_{0: t}-x_{0: t}^{i}\right) d x_{0: t-1}$

where $x_{0: t}^{i}$ is the state trajectory and $y_{1: t}$ are the measurements up to time $t$. The simplest implementation of this algorithm, the Sequential Importance Re-sampling (SIR) particle filter, updates the weights using the likelihood of $y_{t}$ as:

$$
w_{t}=w_{t-1} p\left(y_{t} \mid x_{t}\right)
$$

Long-term predictions are used to estimate the probability of failure in a system given a hazard zone that is defined via a probability density function with lower and upper bounds for the domain of the random variable. The probability of failure at any future time instant is estimated by combining both the weights of predicted trajectories and specifications for the hazard zone through the application of the Law of Total Probabilities. The resulting RUL pdf provides the basis for the generation of confidence intervals and expectations for prognosis and can be computed according to (Acuña and Orchard 2018). As detailed in (De Martin et al. 2017), this approach makes use of degradation models that are tuned or their parameters adjusted to compute the current a-priori state of the system, $p\left(x_{t} \mid y_{1: t-1}\right)$, and to perform the iterative calculation that leads to the long term prediction $p\left(x_{t+k} \mid y_{1: t}\right)$. Auto-tuned models are required to describe and follow changes in the degradation process and to describe, in the best possible way, the process and measurement noise. So, in order to match the model behavior to the real system, as closely as possible, one timedependent tunable model is used.

\subsection{Solution 01 - separated inputs approach}

The first solution to the PHM problem proposed in this paper is to treat the filter clogging process and the actuators wear as two separate faults, to be evaluated through features obtained through sensors pertaining each component without interaction with other subsystems. This solution finds both practical and PHM justifications. Typically, several companies participate in the definition of the complete hydraulic system and there can be an interest for each participant to provide its own, independent and systemagnostic solution to the faults possibly affecting their products. A shown in Fig.9, this solution would require the introduction of "local" intelligent systems, used to collect and process the required data, communicating directly with the central Vehicle Usage and Health Monitoring System (UHMS).

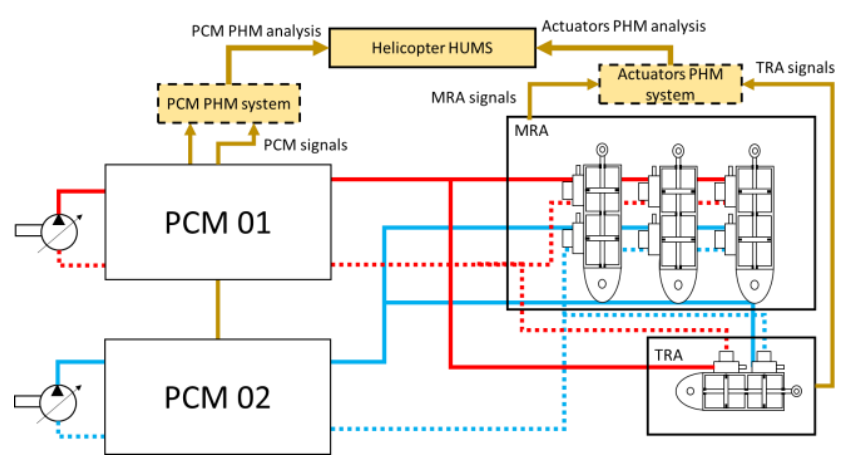

Fig.9. Solution 01 - PHM architecture.

Moreover, signals measured physically close to the fault usually carry more significant information on the fault itself. To best assess the health status of both the filter and the actuators, additional sensors are however needed. Starting from the early results provided in (De Martin et al. 2019), a virtual flow-rate sensor is added to the high-pressure line downstream the filter. The virtual sensor is made of a calibrated hydraulic resistance complete with a differential pressure sensor; the flow-rate is hence derived as a function of the off-line mapping of the calibrated orifice performances. In a similar vein, differential pressure sensors are placed within the actuators' chambers afferent to the same hydraulic line. After a lengthy process of feature selection, where candidates were ranked and compared according to metrics such as correlation with the fault size, signal-to-noise ratio and accuracy, we defined the features $F_{f o_{1}}$ and $F_{A w_{1}}$, respectively for the filter clogging and the actuators wear as,

$$
\begin{aligned}
& F_{f, o_{1}}=\left|\frac{\widehat{\Delta P}_{f}}{\hat{Q}_{f}}\right| \frac{\mu_{40}}{\mu(T)} \\
& F_{A w_{1}}=\left|\frac{\hat{x}_{s, i}}{\widehat{\Delta P}_{a, i}}\right|
\end{aligned}
$$

Where $\widehat{\Delta P}_{f}$ and $\widehat{Q}_{f}$ are the signals measuring pressure drop across the filter and the flowrate across it, all conditioned through a low-pass filter with bandwidth around $0.5 \mathrm{~Hz}$ to cut high-dynamics effects due to the irregular flow-rate request and the capacitive behavior of the short pipe lines within the PCM. $\mu_{40}$ and $\mu(T)$ are instead the expected fluid viscosity at $40^{\circ} \mathrm{C}$ and the expected fluid viscosity at the fluid temperature $T$, which value is measured within the tank. Similarly, $\widehat{\Delta P}_{a, i}$ and $\hat{x}_{s, i}$, are the pressure drop across two adjacent chambers within each actuator, while is the servovalve spool position; as for the signals used to compute the filter clogging feature, even these measurement are conditioned to limit the effect of quick transients over the feature behavior. As shown in Fig.10, both features works fairly well in addressing the respective faults; with the feature related to the filter clogging exhibiting a nonlinear behavior which is directly dependent on the nonlinearity of Eq.(15) and on the different weight that each 
terms of such equation exhibit as the degradation progresses.
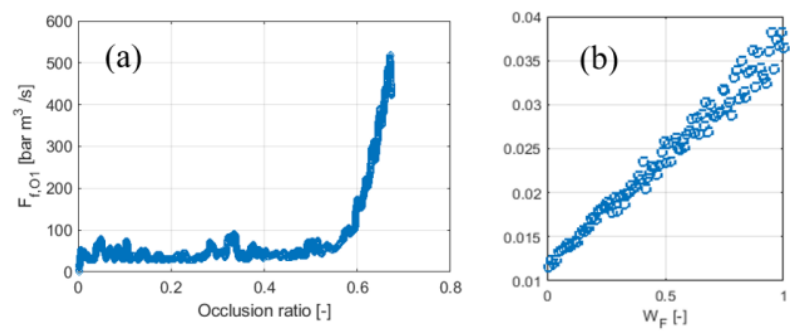

Fig.10. Solution 01 - features behavior (a) filter clogging (b) actuator leakages.

\subsection{Solution 02 - integrated approach without additional sensors}

The first alternative solution presented in this paper leverage the IVHM approach under the assumption that the system owner has access to all of the signals typically present in systems similar to the case study. In this solution, a central intelligent system is in charge of analyzing signals or features coming from each subsystem and performing PHM analysis as shown in Fig.11. In this case, the leakage within each actuator is still detected as in solution 01, but an alternative take on the health monitoring process for the filter clogging is proposed.

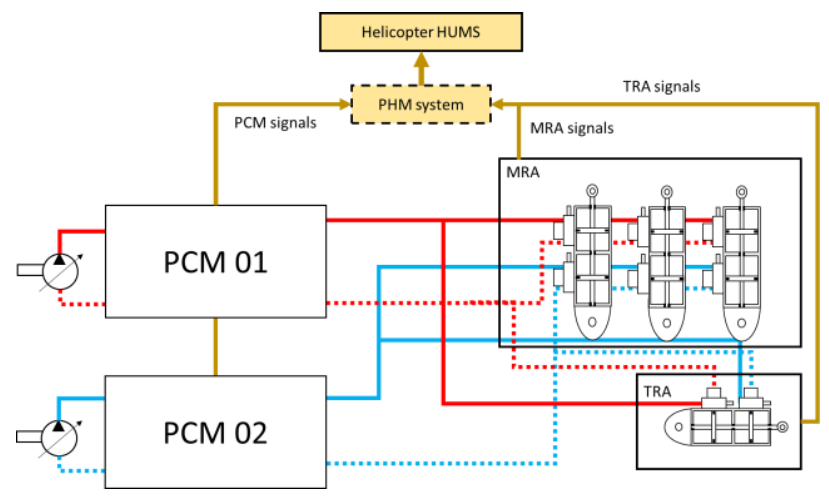

Fig.11. Solution $02-$ PHM architecture.

In this solution, no additional sensors are needed within the PCM, as the flowrate passing through the filter can be roughly estimated through the estimates of the speed signals of each actuator obtained differentiating in time the position signals used for control purposes. Defining with $A_{T M R, i}$, the average effective area of the $i$-th actuator, the feature defined in Eq. (19) becomes,

$$
F_{f, o_{2}}=\left|\frac{\widehat{\Delta P}_{f}}{\sum_{i=1}^{4} A_{T M R, i} \hat{\bar{x}}_{l}}\right| \frac{\mu_{40}}{\mu(T)}
$$

Where is the estimated speed conditioned through a lowpass filter to avoid the influence of quick dynamics and fluid compressibility at the actuator level. As shown in Fig.12, the feature computed though this approach is less accurate than the one obtainable through the signals used in the first solution, but it remains viable for PHM.

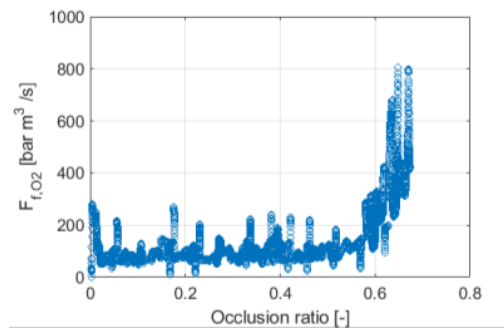

Fig.12. Solution 02 - feature behavior (filter clogging).

\subsection{Results and comparisons}

Both solutions were tested against the same data-set for fault detection and prognosis. Since filters tends to jam before the wear of the actuator seals becomes significant to the system behavior, we focused our attention on the filter clogging. At first, the simple data-driven fault detection technique is applied. Hence the particle filter routines are employed; the particle filter algorithm estimates the defect size (i.e., the percentage of occluded volume within the filter, or the worn volume for the actuators seals) and predict its evolution in time. As shown in Fig.13, the first solution is able to detect the clogging of the high-pressure filter earlier than the second option, with an average clogging ratio at detection, computed over ten degradations histories, of 54\% against $57 \%$. Similar considerations carry to the evaluation of the prognostic performance, were the first solution provides a longer Prognostic Horizon (150 hours against 122 hours, if we consider a 100\% confidence and unitary value of risk) and higher accuracy metric (78.5\% RA against $75.2 \% \mathrm{RA})$. The definition of both metrics is provided in (Saxena et al. 2008). Solution 02 remains however viable and provide the only possible solution for the PHM problem under analysis that does not need the introduction of a dedicated sensor within the PHM. An example of the prognostic output for a non-accelerated clogging of the high-pressure filter is provided in Fig. 14.
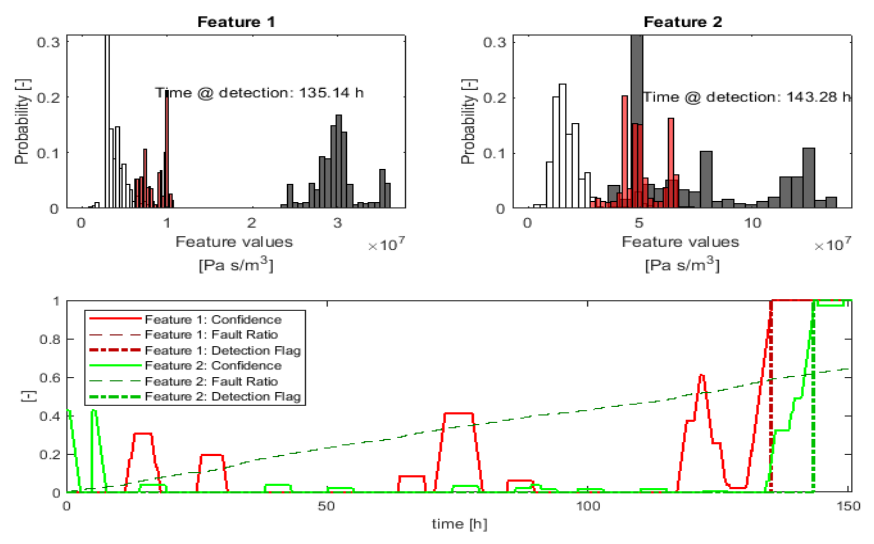

Fig.13. Fault detection against accelerated fault progression. 

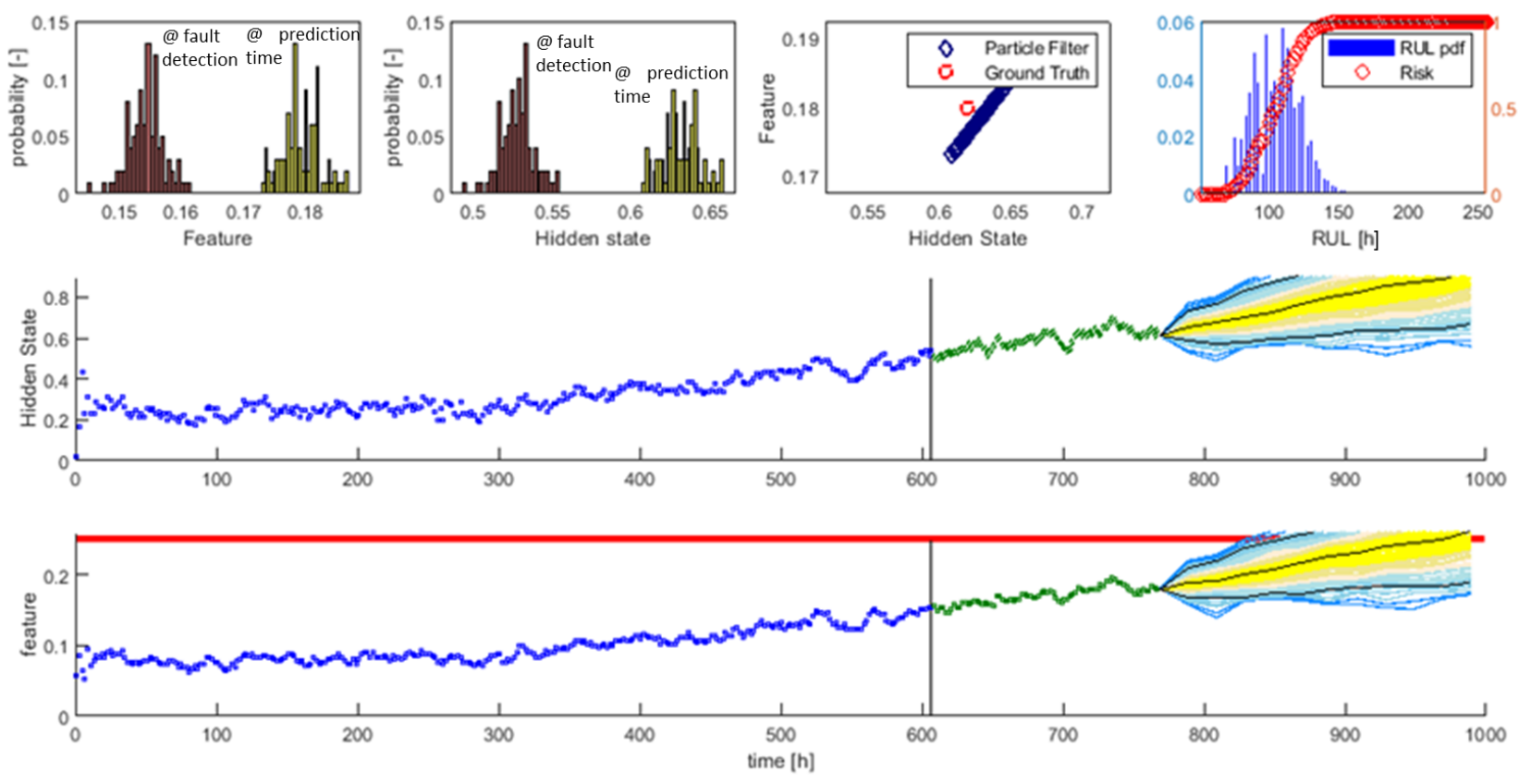

Fig.14. Prognosis for non-accelerated fault progression (Solution 01).

\section{TOWARDS A MORE INTEGRATED APPROACH}

Results described in Section 6 suggests that a more integrated approach to PHM issues is a viable solution to overcome the lack of dedicated sensors in some subsystems; in the case study, a more integrated approach could lead to the detection and prognosis of the filter clogging even in absence of a direct measure of the supply flow-rate sent from the PCM to the flight control actuators. As previously mentioned, the filter clogging process is mainly due to the wear within the actuators and the pump; however, no mention of a health monitoring system dedicated to the pump itself as been introduced so far. Moving toward a more integrated approach, as depicted in Fig.15, allows however to obtain an interesting level of information on the pump wear even without the definition of a dedicated PHM system.

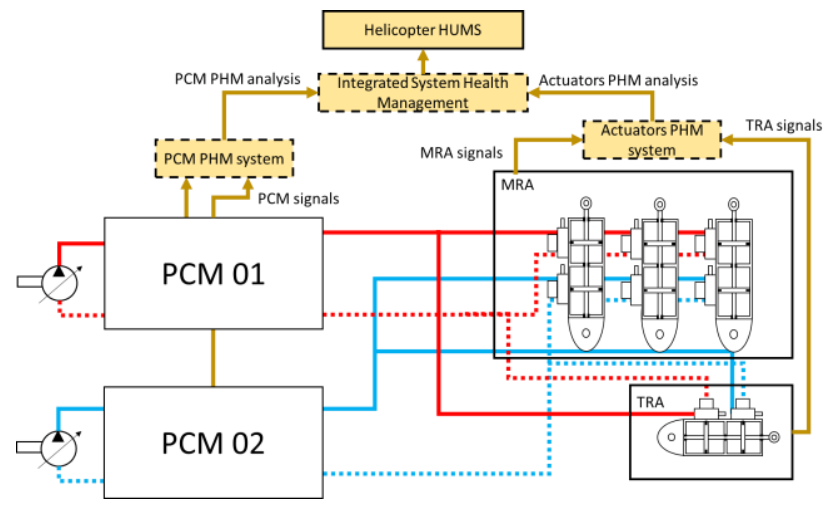

Fig.15. More integrated architecture
In this new PHM architecture, information coming from dedicated PHM functions are not directly sent to the HUMS; instead, an intermediate "Integrated System Health Management" function is inserted to fuse information coming from several local PHM systems, analyze them and provide additional diagnostic and prognostic functionalities.

The overall volume of deposit in the high-pressure filter of the Hydraulic System $01 V_{w, H P F}$ can be approximated as,

$$
V_{w, H P F}=V_{w, T M R}+V_{w, p}-V_{w, L P F}+V_{o p}-o\left(V_{w}\right)
$$

Where $V_{w, T M R}$ is the overall volume of worn material produced in the chambers of the flight control actuators pertaining to the Hydraulic System 01, $V_{w, p}$ is the volume of worn material produced within the pump, $V_{w, L P F}$ is the deposit in the return channel, $V_{o p}$ is the worn volume in the utilities and $o\left(V_{w}\right)$ is the small fraction of debris which size is too small to be kept by any of the filters. Neglecting $V_{o p}$, the overall volume of worn material produced within the pump pertaining one $\mathrm{PCM}$ is,

$$
V_{w, p}=V_{w, H P F}-V_{w, T M R}+V_{w, L P F}+o\left(V_{w}\right)
$$

The volumes $V_{w, H P F}$ and $V_{w, L P F}$ can be directly computed as proportional to the filter clogging ratio estimate provided by the particle filter applied to feature $F_{f, o_{1}}$ or $F_{f, o_{2}}$. In a similar vein, volume $V_{w, T M R}$ can be estimated by combining the hidden states estimates provided by the particle filter algorithms working on the actuators' leakages. Results are encouraging, as depicted in Fig.16, where the application of such considerations to an accelerated wear scenario is represented. 


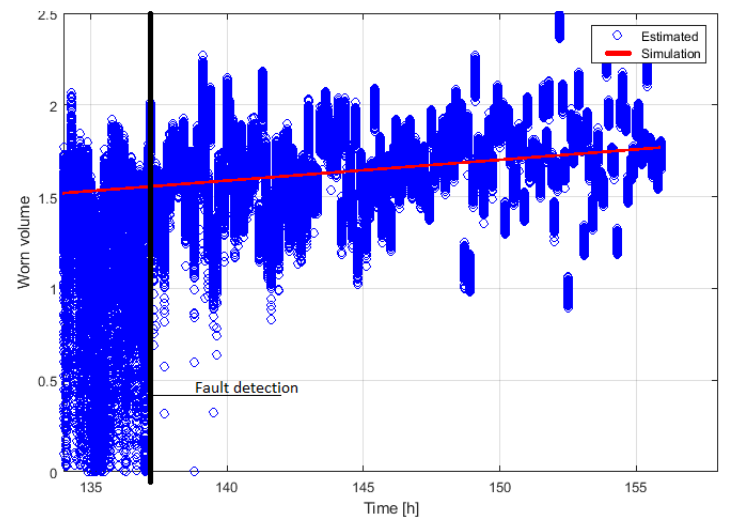

Fig.16. Estimate of the worn material produced by the pump.

The system is supposed to be operative after the fault detection routine observe the filter clogging for the first time. This architecture would also lend itself to the prognosis of the pump wear, with the caveat of retaining the wear estimate after the replacement of any filter of the pertaining PCM. In a similar way it is theoretically possible to evaluate the total flow-rate lost due to leakages if the PCM employs the virtual sensor for flow-rate estimate by combining the two approaches described in Section 6. Both possibilities will be addressed in further works.

\section{CONCLUSION AND FUTURE DEVELOPMENT}

The study presented in this paper report the results of the first steps in a wider research activity aimed at the definition of an integrated Health Management system for the hydraulic system of a fly-by-wire helicopter. An in-depth dynamic model of the whole system has been prepared and its mathematical formulation described. Hence, the wear of moving components in the flight control actuators and pumps of the hydraulic system was correlated with the clogging process of the PCM filters. Models for both degradations and their interaction were introduced and discussed as well. Hence two possible approaches for PHM of such faults were presented, where different levels of integration between signals coming from different subsystems were investigated. Results were hence compared. Finally, results coming from this investigation were combined to show how fusing information provided by different PHM algorithms operating on different systems can be used to infer the health status of other devices, in this case the pump wear, which are usually not equipped with enough sensors to build a comprehensive PHM system. Early results are encouraging but require further work in both simulation campaigns and eventually experimental activities. Moreover, additional studies are needed to best define the implementation of such PHM and IVHM functionalities on a real platform (on-board, off-board, a mix of both). Future development will include the study of more degradation modes within each subsystem (pump,
PCM, flight control actuators) and their interaction in the attempt to further exploit the more integrated approach to PCM for flight control systems proposed in this paper.

\section{NOMENCLATURE}

$\alpha \quad$ Angular position of the piston pump shaft

$\beta \quad$ Bulk modulus for the considered fluid

$\mu(T) \quad$ Expected dynamic viscosity of the hydraulic fluid at temperature $T$

$\mu_{40} \quad$ Expected dynamic viscosity of the hydraulic fluid at the reference temperature $\left(40^{\circ} \mathrm{C}\right)$

$\rho_{h} \quad$ Density of the hydraulic fluid

$\Delta P_{f} \quad$ Pressure drop across the considered filter

$\Delta P_{p} \quad$ Pressure drop across the pump

$\Delta P_{v} \quad$ Pressure drop across the considered valve

$\triangle V_{w, H P F}$ Increment of the filtered volume in the highpressure filter

$\triangle V_{w, L P F} \quad$ Increment of the filtered volume in the lowpressure filter

$\omega$ Angular frequency of the pump

$A_{i} \quad$ Thrust area for the $i$-th chamber of the considered actuator

$A_{v} \quad$ Metering area of the considered valve

$C_{d} \quad$ Discharge coefficient of the considered valve

c Viscous friction coefficient for the considered actuator

$c_{h} \quad$ Damping of the actuator's joints

$c_{v} \quad$ Viscous friction coefficient for the considered valve

$F^{*} \quad$ Cracking force of the considered valve

$F_{\text {ext }} \quad$ External force acting on the considered actuator

$F_{f r, i} \quad$ Friction forces for the $i$-th contact point of the considered actuator

$K_{\text {leak }} \quad$ Leakage coefficient

$k_{h} \quad$ Stifness of the actuator's joints

$k_{v} \quad$ Spring stiffness for the considered valve

$m_{v} \quad$ Mass of the moving element for the considered valve

$m_{r} \quad$ Rod mass of the considered actuator

$P_{c} \quad$ Pressure of the piston pump chamber

$P_{i} \quad$ Pressure of the $i$-th chamber of the considered 


$\begin{array}{ll} & \text { actuator } \\ Q_{i} & \text { Pump flow-rate } \\ Q_{i n} & \text { Inlew-rate supplied by the } i \text {-th piston of the pump } \\ Q_{\text {out }} & \text { Outlet flow-rate for the considered piston } \\ Q_{l e a k} & \text { Flow-rate lost due to leakages within the pump } \\ Q_{v} & \text { Flow-rate through the condisdered valve } \\ R e & \text { Reynolds' number } \\ V_{i} & \text { Volume of the } i \text {-th piston of the pump } \\ W_{r} & \text { Weight of the rod for the considered actuator } \\ W_{s} & \text { Weight of the sleeve for the considered actuator } \\ V_{w, i} & \text { Worn volume of material for the } i \text {-th seal } \\ V_{w, H P F} & \text { Volume of material filtered by the high-pressure } \\ V_{w, L P F} & \text { filter } \\ V_{w, p} & \text { Volume of material filtered by the low-pressure } \\ V_{w, T M R} & \text { Worn volume produced by the TMR }\end{array}$

\section{REFERENCES}

Acuña, D. E., and Orchard, M. E. (2018). “A theoretically rigorous approach to failure prognosis." Proceedings of the 10th Annual Conference of the Prognostics and Health Management Society 2018 (PHM18).

Adams, S., Beling, P. A., Farinholt, K., Brown, N., Polter, S., and Dong, Q. (2016). "Condition based monitoring for a hydraulic actuator." Proceedings of the Annual Conference of the Prognostics and Health Management Society, PHM, 2016-Octob, 380-389.

Autin, S., Socheleau, J., Dellacasa, A., De Martin, A., Jacazio, G., and Vachtsevanos, G. (2018). "Feasibility Study of a PHM System for Electro-hydraulic Servoactuators for Primary Flight Controls." Annual Conference of the Prognostic and Health Management Society, 1-19.

Brown, D., Georgoulas, G., Bae, H., Vachtsevanos, G., Chen, R., Ho, Y. H., Tannenbaum, G., and Schroeder, J. B. (2009). "Particle filter based anomaly detection for aircraft actuator systems." IEEE Aerospace Conference Proceedings.

Byington, C. S., Watson, M., and Edwards, D. (2004). "Data-driven neural network methodology to remaining life predictions for aircraft actuator components." IEEE Aerospace Conference Proceedings, 6, 3581-3589.

Carman, P. C. (1997). "Fluid flow through granular beds." Process Safety and Environmental Protection: Transactions of the Institution of Chemical Engineers, Part $B$.

Eker, O. F., Camci, F., and Jennions, I. K. (2016). "Physicsbased prognostic modelling of filter clogging phenomena." Mechanical Systems and Signal Processing.

Ergun, S. (1952). "Fluid flow through packed columns." Chemical Engineering Progress.

Frölich, D., Magyar, B., and Sauer, B. (2014). "A comprehensive model of wear, friction and contact temperature in radial shaft seals." Wear, Elsevier, 311(1-2), 71-80.

Idelchik, I. E., and Fried, E. (1986). Handbook of hydraulic resistance: Second edition.

De Martin, A., Dellacasa, A., Jacazio, G., and Sorli, M. (2018). "High-Fidelity Model of Electro-Hydraulic Actuators for Primary Flight Control Systems." Proceedings of the 2018 Bath/ASME Symposium on Fluid Power and Motion Control FPMC2018 September 12-14, 2018, University of Bath, United Kingdom, V001T01A058.

De Martin, A., Jacazio, G., and Sorli, M. (2017). “Enhanced Particle Filter framework for improved prognosis of Electro-Mechanical flight controls Actuators." 3rd European Conference of the Prognostic and Health Management Society, 1-10.

De Martin, A., Jacazio, G., Sorli, M., and Surdo, V. (2019). "Preliminary analysis on an IVHM approach for prognosis of high-pressure filters for hydraulic Power Control Modules of helicopters." MATEC Web of Conferences, 304, 04017.

Nesci, A., De Martin, A., Jacazio, G., and Sorli, M. (2020). "Detection and Prognosis of Propagating Faults in Flight Control Actuators for Helicopters." Aerospace, $7(3), 20$.

Orchard, M. E., and Vachtsevanos, G. J. (2009). "A particlefiltering approach for on-line fault diagnosis and failure prognosis." Transactions of the Institute of Measurement and Control.

Roemer, M. J., Byington, C. S., Kacprzynski, G. J., Vachtsevanos, G., and Goebel, K. (2011). "Prognostics." System Health Management: With Aerospace Applications.

Saxena, A., Celaya, J., Balaban, E., Goebel, K., Saha, B., Saha, S., and Schwabacher, M. (2008). "Metrics for evaluating performance of prognostic techniques." 
2008 International Conference on Prognostics and Health Management, PHM 2008.

Smith, M., Kloda, J., and Friel, J. (2014). "Hydraulic system internal and external leakage diagnostics." Proceedings of the American Helicopter Society 70th Annual Forum, Montreal, Canada.

Vachtsevanos, G., Lewis, F., Roemer, M., Hess, A., and $\mathrm{Wu}$, B. (2007). Intelligent Fault Diagnosis and Prognosis for Engineering Systems. Intelligent Fault Diagnosis and Prognosis for Engineering Systems.

Vianna, W. O. L., and Malere, J. P. P. (2014). "Aircraft hydraulic system leakage detection and servicing recommendations method." PHM 2014 - Proceedings of the Annual Conference of the Prognostics and Health Management Society 2014. 